\title{
Nasopalatine duct cyst: report of 52 cases in a retrospective epidemiological study
}

\section{Francesco Cecchetti ${ }^{1}$, Matacena Giada ${ }^{2}$, Cicciù Marco $^{3}$, Francesco Germano ${ }^{1}$, Claudio Arcuri ${ }^{1}$, Bramanti Ennio ${ }^{2}$}

${ }^{1}$ Department of Periodontics, University of Rome (Tor Vergata), Italy

${ }^{2}$ Department of Experimental Medical-Surgical and Oral Dental Sciences, University of Messina, Italy

${ }^{3}$ Department of Human Pathology, University of Messina, Italy

\section{Email address:}

enniobramanti@hotmail.it(E. Bramanti), francesco.cecchetti@tin.it(F. Cecchetti)

\section{To cite this article:}

Cecchetti Francesco, Matacena Giada, Cicciù Marco, Francesco Germano, Claudio Arcuri, Bramanti Ennio. Nasopalatine Duct Cyst: Report of 52 Cases in a Retrospective Epidemiological Study. Journal of Surgery. Vol. 1, No. 2, 2013, pp. 14-17.

doi: $10.11648 /$ j.js. 20130102.12

\begin{abstract}
Introduction. Nasopalatine duct cysts (NPDCs) are the most common developmental epithelial non-odontogenic cysts of the upper maxilla. Their etiological origin is still uncertain; NPDCs are often asymptomatic and are usually found randomly through local radio-diagnostic investigations carried out for other reasons. Main body. The aim of this investigation is to analyze and discuss the etiology, differential diagnosis, clinic-pathological characteristics of these lesions as well as to report the relative frequency and distribution of nasopalatine duct cysts in general population (NPDCs) with a literature's review on the topic. The retrospective study was carried out using 52 clinical cases, with histopatological confirmation for NPDC, radiographs and oral photographs. Data included age and gender of the patient, radiographic findings, etiological factors, treatment, and prognosis of NPDC. Few surgical consideration are discussed. The study results report a NPDCs' incidence of $2.39 \%$ and a male predilection with a 2:1.25 ratio. No statistically significant correlation was observed between the size of the lesion and patient's gender. Lesions were usually asymptomatic (73\%). All cysts were located in the anterior maxillary midline region. Panoramic X-rays and computed tomography was used to identify the lesion. Surgical treatment was performed under local anesthesia including the dissection and the removal of the cyst, usually adopting a palatine approach with an enveloping flap from 1.4 to 2.4 . Relapse occured in $15.4 \%$ of the cases. Conclusions. The etiology of NPDC is unclear and a male predilection was observed. The histological analysis of cystic lesions was fundamental for the final diagnosis. Simple surgical resection was recommended, followed by clinical and radiological control to ensure the correct and complete resolution of the case.
\end{abstract}

Keywords: Nasopalatine Duct Cyst, Non-Odontogenic Cyst, Maxillary Cyst

\section{Introduction}

Jaw cyst is a pathological intra-osseous cavity lined by a membrane and may contain liquid or semisolid material[1]. Cysts in maxillar, mandibular and perioral regions widely change in distribution, characteristics, histogénesis, incidence, behavior and treatment[2].

Authors have classified cysts in odontogenic, non-odontogenic cysts, and pseudocysts. Odontogenic cysts originate from odontogenic epithelium and arise in the tooth-bearing regions of the jaws. Non-odontogenic cysts probably derive from the proliferation of non-odontogenic epithelial remnants that are trapped along fusion lines during embryogenesis of the cephalic district. Pseudo-cysts differ from a real cyst because of the absence of the epithelial membrane[3]. The nasopalatine duct cyst (NPDC) is an intraosseous developmental cyst of the midline of the anterior palate. Studies have reported that the nasopalatine duct cysts (NPDCs) are the most common non-odontogenic, developmental and epithelial cysts of the oral cavity corresponding to the $1 \%$ of all maxillary cysts [4-5] and the $26.6 \%$ of all non-odontogenic cysts [6].

The maximum incidence is between 40 and 60 years old and these lesions are more frequent in males than in females, with a 3:1 ratio[5]. It's not completely clear whether NPDCs are more common in Caucasians, Asians or Negroes $[4,6]$ because of a lack of representative epidemiological studies; NPDCs are uncommon in children, 
especially in Caucasian children [7].

This lesion (NPDC) was firstly described by Meyer [8] in 1914 as a "paranasal sinus". In the past NPDC was known as "anterior middle cyst", "anterior middle palatine cyst", and "incisor duct cyst" and was regarded as a fissural cyst $[4,9]$ which originated from the epithelium trapped during the fusion of the embryological process. At present, these concepts have been partially discarded.

Nowadays according to the classification of the World Health Organization (WHO), these lesions are considered as non-odontogenic cysts of the upper maxilla which originate from embryologic remnants of nasopalatine duct and frequently develope in the midline of anterior maxilla close to the incisive foramen[10-11]. NPDC is a slow-growing cyst and can habitually and spontaneously release a mucinous liquid[12].

NPDCs' origin is uncertain[13]. Many possible etiologic factors have been proposed: local trauma during mastication or ill fitting dentures, bacterial infection, spontaneous proliferation, mucous retention of adjacent minor salivary glands, blockage of glandular ducts, racial or genetic factors[11,14]. Although pathogenesis of this lesion is still uncertain, many authors suggest the theory of spontaneous cystic degeneration and proliferation[4,13].

Histological features of cystic epithelium vary according to the location involved (palatine, nasal or intermediate). A squamous cell epithelium is often observed, though a ciliary respiratory type epithelium can be seen when lesion is positioned higher or nasally[4] . Lesions are normally asymptomatic and frequently constitute casual radiological findings[8]. Sometimes (17\% of cases) patients report pain due to the compression of adjacent structures, especially when there is a process of over-infection or in patients who wear dentures that compress the zone. Usually early symptoms could appear for the caudal location of the cyst[5].

Inflammatory process ( $46 \%$ of cases) rarely produces facial asymmetry, because the growth or expansion is intraorally (palatine) [4].

The most commonly reported clinical symptoms are: salty taste and swelling on the anterior part of the palate[11,13].

In rare cases patients have complained a burning sensation in the anterior part of the maxilla that occasionally radiates into the bridge of the nose and the orbits [15].

Radiologically, NPDCs appear as a well delimited, rounded or heart-shaped radiotransparency circumscribed to the upper interincisal midline [Fig. 1].

The differential diagnosis is established with the following conditions: an enlarged nasopalatine duct, a central giant granuloma, an upper central incisor root cyst, a supernumerary tooth follicular cyst (normally mesiodens), primordial cyst, nasoalveolar, cyst, osteits with palatal fistulization, a bucconasal and or buccosinusal communication $[4-5,12]$. Treatment in all cases involves complete surgical removal as soon as possible after diagnosis. It was reported a relapse rate of the $30 \%[4]$.

The purpose of this epidemiological and descriptive study is to analyze, consolidate and organize available information regarding NPDCs with our clinical experience and epidemiological data upon 52 cases and with a review of the literature.

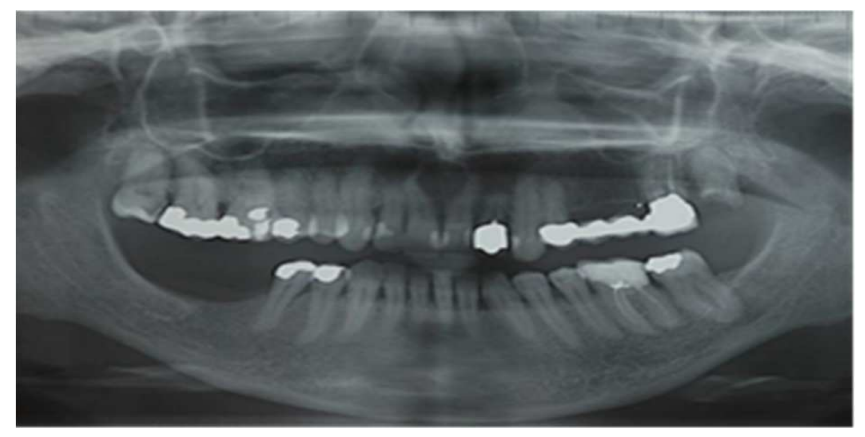

Fig 1. Radiological Aspect

\section{Main Body}

A retrospective observational study was made in a 14-year-period (from 1998 to 2012) selecting, among 2175 patients evaluated for surgery treatments of cystic lesion, 52 cases with histopathological confirmation of NPDC. The study included patients treated in the Oral Surgery department of "S. Giovanni Calibita" hospital Fatebenefratelli, Isola Tiberina (University of Rome, Tor Vergata). For each case, the following data of the lesion history were compiled: patient's age and gender, cyst possible etiology, shape and dimensions of the lesion, radiographic appearances, symptoms, histology, surgical treatments and course. Panoramic X-rays were obtained in all cases and computed tomography (CT) scans have been requested only in some cases[Fig. 2].

Surgical treatment was executed under local anaesthesia and comprised the dissection and removal of the cyst usually adopting a palatine approach. An enveloping flap from 1.4 to 2.4 dental elements was generally prepared[Fig. 3].

Biopsies were surgically excised and the samples obtained were sent to the Laboratory of Oral Pathology ("S. Giovanni Calibita" hospital - Fatebenefratelli, Isola Tiberina) in $10 \%$ formalin solution after staining with hematoxylin-eosin, for histological studies [12]. They were after recorded as nasopalatine duct cysts.

In the histological evaluation, cyst epithelium type and the cystic wall features were reviewed [Fig. 4]. Out of 2175 patients evaluated for surgery treatments of cystic lesion in the period of this study (14 years), fifty-two cases were diagnosed as NPDC. The majority of patients were males (32 patients, 61.5\%) while 20 patients (38.5\%) were females, registering a $\mathrm{M}: \mathrm{F}$ ratio of $2: 1.25$. There was no significant difference in age at the time of diagnosis between males and females $(\mathrm{P}=0.082)$. The overall mean age at the time of diagnosis was 54.5 years old and it was 
higher in females (mean value 58.6 years old) than in males (mean value 50.4 years old)[Tab. 1]. Fifteen patients presented antecedent diseases of interest: 6 arterial hypertension, 5 diabetes mellitus type II, 3 idiopatic osteoporosis, 1 Hashimoto's thyroiditis [Tab. 2].

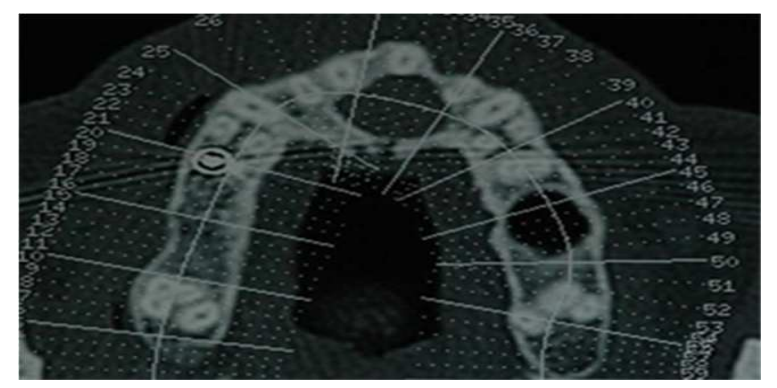

Fig 2. TC Dentascan Axial Wiew

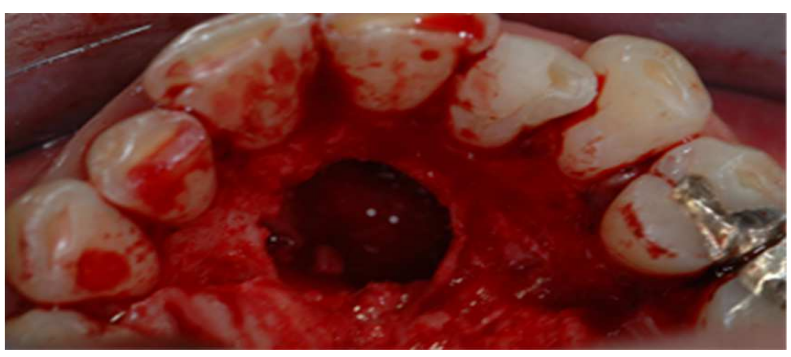

Fig 3. Flap design

Regarding toxic habits: only seven patients were smokers, nobody reported alcohol consumption [Tab. 2]. For two patients the nasopalatine duct could have become infected via the nasal route, since they presented chronic bacterial rhinitis without symptoms at the time of diagnosis.

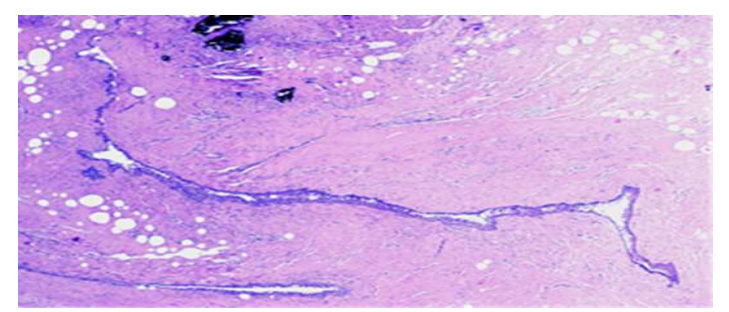

Fig 4. Histological Section following ematoxylin-eosin staining

The vitality of the adjacent teeth (permanent upper central incisors) was preserved in the $59.6 \%$ of the cases $(\mathrm{n}=31)$ [Tab. 3]. 38 patients were completely asymptomatic $(73 \%)$ [Tab. 2], 9 subjects $(17.3 \%)$ showed local inflammation, while the remaining $5(9.7 \%)$ reported pain and ulceration

Tab 1. Age and gender of patients with NPDCs

\begin{tabular}{ll}
\hline Gender & Mean Age \pm Standard Deviation \\
\hline Male $(\mathrm{n}=32)$ & $50.4 \pm 8.6$ \\
Female $(\mathrm{n}=20)$ & $58.6 \pm 11.5$ \\
Total subjects $(\mathrm{n}=52)$ & $54.5 \pm 10.1$ \\
\hline
\end{tabular}

Produced by dentures. The position of the NPDCs was mostly superficial or palatine (44 cases) [Tab. 2], while in only 8 cases the lesions were located more deeply or in the nasal region. For these patients a computed tomography scan was requested in order to establish precisely the NPDCs position. In all cases, treatment consisted in a complete surgical removal of the cystic lesion through a combination of cold scalpel and piezosurgery (PIEZOSURGERY ${ }^{\circledR}$ touch starter Mectron), with careful dissection of the neurovascular bundle. In 51 cases (98\%) a palatine approach was used [Tab. 2], while a vestibular access was preferred only in a single case $(2 \%)$. The patients operated upon the palatine approach, after surgery reported discomfort due to important swelling, pain and tenderness in the anterior region of the hard palate (submucosal hematoma) during the first two postoperative weeks. The follow-up mean duration was two years, until correct ossification of the surgical zone was confirmed by $\mathrm{X}$-ray study. Following surgical exeresis, 44 lesions (84.6\%) healed completely after the first operation, while the remaining 8 cases $(15.4 \%)$ suffered relapse after 3-year-follow-up [Tab. 2]. This situation required a second intervention (removal of the NPDC with ligation of the nasopalatine neurovascular bundle), after which complete healing was reached. Our study resulted in agreement with the literature for NPDCs' male predilection and mean age of incidence [Tab.1][2,4,6].

Although the etiology of these lesions is not clear, in addition to the hypothesis of spontaneous proliferation from embryonic tissue remains, other possible etiologies have been proposed, such as prior trauma, poorly fitting dentures, local infection, genetic and racial factors. Most of the cysts were asymptomatic (38 of the 52 patients with NPDC in our study), and constituted casual findings. Any clinical manifestations that may appear were attributable to inflammation, in which case pain, itching, ulceration, local infection and/or fistulization were observed $[8,12]$.

The diagnosis of NPDC was based upon clinical history, clinical exploration and complementary tests. Radiological exams (panoramic X-rays, periapical and occlusal X-rays and computed tomography) were necessary to correctly diagnose NPDCs [16].

Radiologically, lesions appeared as a well delimited radiotransparency, measuring often $1-2 \mathrm{~cm}$ in diameter [16-17]. The X-ray image was predominantly rounded, ovoid or heart-shaped [Figs. 1,2].

The nasopalatine neurovascular bundle is a delicate and highly vascularized structure giving rise to profuse bleeding if inadvertently sectioned during surgery. We therefore considered that the piezosurgery inserts offered adequate safety in these surgical procedures, and used in our NPDCs exeresis, reduced post-operative pain and edema. Paresthesia of the anterior palatal zone was a rare complication consequent to the removing of nasopalatine nerve endings along with the membrane of the cyst [12]. In this study we recorded three cases $(5.8 \%)$ of palatal paresthesia [Tab. 2]. The histological analysis of NPDCs revealed squamous cell epithelium 32 cystic lesions $(61.5 \%)$ 
though in the $38.5 \% \quad(n=20)$ of cases squamous cell epithelium was combined with other types of epithelium such as ciliary cylindrical cells, especially in deep-lying or nasal cysts.

Tab 2. Epidemiological data recorded in 52 patients with NPDC

\begin{tabular}{lcc}
\hline & Percentage(\%) & $\begin{array}{c}\text { Number of } \\
\text { cases(n) }\end{array}$ \\
\hline Incidence of NPDCs & $2.39 \%$ & $52 / 2175$ \\
Patients with other diseases & $28.8 \%$ & $15 / 52$ \\
Smokers & $13.46 \%$ & $7 / 52$ \\
Alcohol & $0 \%$ & $0 / 52$ \\
Asympomatic NPDC & $73 \%$ & $38 / 52$ \\
Vitality of adjacent teeth & $59.6 \%$ & $31 / 25$ \\
Superficial or palatal NPDC & $84.6 \%$ & $44 / 52$ \\
localization & $98 \%$ & $51 / 52$ \\
Surgical palatine approach & $15.4 \%$ & $8 / 52$ \\
Relapse & $5.8 \%$ & $3 / 52$ \\
Palatal paresthesia & & \\
\hline
\end{tabular}

\section{Conclusions}

Nasopalatine duct cyst are the most common non-odontogenic cysts of the jaws.

NPDCs had to be distinguished from other frequent anterior maxillary lesions.

Men were most susceptible to NPCDs than women.

Adjacent teeth vitality testing or radiodiagnostic images were not enough for a final diagnosis. The definitive diagnosis could only be performed after histological analysis. Therefore, in 2175 subjects evaluated in the period of the study (14 years), the incidence of the NPDC was $2.39 \%$. The cystic epithelium varied considerably both among different cases and also within the same cyst.

The treatment of choice was surgical exeresis of the cyst, although some authors proposed marsupialization of the largest NPDCs [1,4].

A simple surgical resection was recommended and it should be followed by a clinical and radiological control in order to ensure a complete correct resolution of the lesion .

It was registered a lower relapse rate $(15.4 \%)$ compared to that reported in literature $(30 \%)$ after a follow-up of 3 years.

In conclusion, we could consider NPDC as a medium rare pathology, which is easily solved through a simple, little risky and minimally invasive surgical exeresis. It is important to make an early diagnosis, which is often accidental, due to the frequent absence of symptoms of these lesions.

\section{References}

[1] Reyes Velázquez JO, Palemón HSC, Jiménez Cruz N, Martínez CLE. Quiste del conducto nasopalatino: reporte de un caso. Med Oral 2006; 8(4): 168-171.

[2] Manor E, Kachko L, Puterman MB, Szabo G, Bodner L. Cystic Lesions of the Jaws - A Clinicopathological Study of 322 Cases and Review of the Literature. Int. J. Med. Sci. 2012; 9(1): 20-26

[3] Summers GW. Jaw Cists: diagnosis and treatment. Head \& Neck. 1979; 1(3): 243-258.

[4] Escoda Francolí J, Almendros Marqués N, Berini Aytés L, Gay Escoda C. Nasopalatine duct cyst: Report of 22 cases and review of the literature. Med Oral Patol Oral Ci Bucal. 2008; 1;13(7): E438-43.

[5] Swanson KS, Kaugars GE, Gunsolley JC. Nasopalatine duct cyst: an analysis of 334 cases. J Oral Maxillofac Surg. 1991; 49(3): $268-71 .$.

[6] Johnson NR, Savage NW, Kazoullis S, Batstone MD. A prospective epidemiological study for odontogenic and non-odontogenic lesions of the maxilla and mandible in Queensland. Oral Surg Oral Med Oral Pathol Oral Radiol 2013; 115: 515-522.

[7] Velesquez MT, Mason C, Coonar H, Bennett J. A nasopalatine cyst in an 8-year-old child. Int J Ped Dent. 1999; 9: 123-127.

[8] Meyer AW. A unique supernumerary Paranasal sinus directly above the superior incisors. J Anato My 1914; 48:118-29.

[9] Berlove IJ. Anterior Median palatine cyst. New York State Dental Journal 1956; 26:381-384.

[10] Ely N, Sheehy E, McDonald F. Nasopalatine duct cyst: A case report. Int J Ped Dent 2001; 11:135-7.

[11] Hegde RJ, Shetty R. Nasopalatine duct cyst. J Indian Soc Pedod Prev Dent 2006; 24:31-2.

[12] Gnanasekhar JD, Walvekar SV, Al-Kandari AM, Al-Duwairi Y. Misdiagnosis and mismanagement of nasopalatine duct cyst and its corrective therapy. A case report. Oral Surg Oral Med Oral Pathol Oral Radiol Endod. 1995; 80(4): 465-470.

[13] Allard RH, Van der Kwast WA, Van der Waal I. Nasopalatine duct cyst. Review of the literature and report of 22 cases. Int J Oral Surg. 1981; 10(6): 447-61.

[14] Staretz L, Brian B. Well defined radiolucent lesion in the maxillary anterior region. J Am Dent Assoc 1990;120:335-6.

[15] Vasconcelos RF, de Aguiar F, Castro WH, Cavalcanti de Araùjo V, Mesquita RA. Retrospective analysis of 31 cases of nasopalatine duct cyst. Oral Disease. 1999; 5: 325-328.

[16] Spinelli HM, Isenberg JS, O’Brien M. Nasopalatine duct cysts and the role of magnetic resonance imaging. J Craniofac Surg 1994; 5:57-60.

[17] Staretz LR, Brada BJ, Schott TR. Well-defined radiolucent lesion in the maxillary anterior region. J Am Dent Assoc $1990 ; 120: 335-6$ 